\title{
THE HAUSDORFF MEAN OF A FOURIER-STIELTJES TRANSFORM
}

\author{
CONSTANTINE GEORGAKIS
}

(Communicated by J. Marshall Ash)

\begin{abstract}
It is shown that the integral Hausdorff mean $T \hat{\mu}$ of the FourierStieltjes transform of a measure on the real line is the Fourier transform of an $L^{1}$ function if and only if $T \hat{\mu}$ vanishes at infinity or the kernel of $T$ has mean value zero. Also a sufficient condition on the kernel of $T$ and a necessary and sufficient condition on the measure is established in order for $-i \operatorname{sign}(x) T \hat{\mu}(x)$ to be the Fourier transform of an $L^{1}$-function. These results yield an improvement of Fejer's and Wiener's formulas for the inversion of Fourier-Stieltjes transforms, the uniqueness property of certain generalized Fourier transforms, and a generalization of the mean ergodic theorem for unitary operators.
\end{abstract}

Let $M(R)$ be the space of complex bounded regular Borel measures on the real line $R$ and $\hat{\mu}(x)$ be the Fourier-Stieltjes transform of a measure $\mu$ in $M(R)$,

$$
\hat{\mu}(x)=\int_{R} e^{-i x t} d \mu(t), \quad x \in R .
$$

We consider the integral Hausdorff mean $T \hat{\mu}$ generated by a Borel measurable kernel $\psi$ in $L^{1}(R)$, which is defined for $x \in R$ by

$$
T \hat{\mu}(x)=\int_{R} \hat{\mu}(x s) \psi(s) d s=\frac{1}{|x|} \int_{R} \psi\left(\frac{y}{x}\right) \hat{\mu}(y) d y,
$$

for $x \neq 0$, and $T \hat{\mu}(0)=\hat{\mu}(0) \int_{R} \psi(s) d s$. When the kernel $\psi$ is the characteristic function of $[0,1], T \hat{\mu}$ reduces to the integral arithmetic average of $\hat{\mu}$ over $[0, x]$.

The summability properties of $T$ are well known [8, pp. 275-278]. The continuity properties and the spectrum of $T$ as a bounded operator on $L^{p}(R)$ have been studied by Schur [14], Hardy, Littlewood, and Polya [9], Rhoades [12], Fabes, Jodeit, and Lewis [3], and Leibowitz [11]. Goldberg in [5, 6] studied the properties of the transformation $T$ on the Fourier transform for the Lebesgue class $L^{p}(R)$ for $1<p \leq 2$.

The objective here is to determine the Fourier analytic properties of $T$ on the class of Fourier-Stieltjes transforms. Theorems 1 and 3 are the main results,

Received by the editors February 18, 1991 and, in revised form, March 22, 1991; presented at the annual meeting of the AMS held in January 1991, in San Francisco.

1991 Mathematics Subject Classification. Primary 42A38; Secondary 47A35, 26D15.

Key words and phrases. Hausdorff mean Fourier-Stieltjes, transform, ergodic, unitary operator. Supported through a research grant from DePaul University, Summer 1989. 
which include the analogue of the results of Goes [7] and Georgakis [4], concerning the arithmetic means of Fourier-Stieltjes coefficients of a measure on the circle for the integral arithmetic average of the Fourier-Stieltjes transform of a measure on the real line. As a consequence, we obtain: (a) certain improved variants of the formulas of Fejer and Wiener for the inversion and quadratic variation of Fourier-Stieltjes transforms (Corollary 1); (b) a strengthened generalization of the mean ergodic theorem for a one-parameter group of unitary operators. Theorem 2 establishes the uniqueness of the generalized Fourier transforms introduced by Sz.-Nagy [14] and studied extensively in [1].

We show that in general $T \hat{\mu}$ is a Fourier-Stieltjes transform and, in particular, $T \hat{\mu}$ is the Fourier Transform of a function in $L^{1}(R)$ if and only if the kernel $\psi$ has mean value zero over $R$, or $T \hat{\mu}$ tends to zero at infinity. Moreover, $-i \operatorname{sign}(x) T \hat{\mu}(x)$ is the Fourier transform of a function in $L^{1}(R)$ if and only if $T \hat{\mu}$ tends to zero at infinity and $x^{-1} \mu((-x, x))$ belongs to $L^{1}(R)$, assuming that the kernel $0 \not \equiv \psi \in L^{1}(R)$ is nonnegative, nonincreasing, bounded, and has support in $[0, \infty)$; the conditions imposed on the kernel $\psi$ for the latter are satisfied by the usual summability kernels including $e^{-s}, e^{-s^{2}}, 1 \backslash\left(1+s^{2}\right)$, and $\alpha(1-s)^{\alpha-1}$ for $\alpha \geq 1$ and $0 \leq s \leq 1$.

The properties of the transformation $T$ depend on those of the transformation $S$ on $M(R)$, which is defined as

$$
S \mu(x)= \begin{cases}\int_{R-\{0\}} \frac{1}{|y|} \psi\left(\frac{x}{y}\right) d \mu(y) & \text { for } 0 \neq x \in R, \\ 0 & \text { for } x=0 .\end{cases}
$$

It should be noted that $S \mu$ is an even or odd function if the corresponding measure $\mu$ is even or odd, that is, $\mu(-E)=\mu(E)$ or $\mu(-E)=-\mu(E)$ for each Borel subset $E$ of $R$, respectively. Furthermore, if $\mu \geq 0$ and the kernel $\psi \geq 0$ is nonincreasing with support on $[0, \infty)$, then $S \mu$ is nonincreasing for $x>0$.

Theorem 1. Let $T \hat{\mu}$ be the Hausdorff mean of the Fourier-Stieltjes transform of a measure $\mu \in M(R)$ whose kernel $\psi \in L^{1}(R)$. Then

(a) $S \mu \in L^{1}(R)$;

(b) $T \hat{\mu}$ is a Fourier-Stieltjes transform, and $T \hat{\mu}(x)=\mu(\{0\}) \int_{R} \psi(s) d s+$ $(S \mu)^{)}(x), x \in R$;

(c) $T \hat{\mu}$ is the Fourier transform of a function in $L^{1}(R)$ if and only if either $\psi$ has mean value zero or $T \hat{\mu}$ tends to zero at infinity, which is equivalent to $\mu(\{0\})=0$.

Proof. Clearly, (c) follows from (a) and (b), since $(S \mu)(x) \rightarrow(x)$ as $|x| \rightarrow \infty$ by the Riemann-Lebesgue theorem. For (a) and (b), we may assume without loss of generality that $\mu$ is nonnegative. Then Fubini's theorem yields

$$
\begin{aligned}
\int_{R} S \mu(x) d x & =\int_{R-\{0\}} d \mu(y) \int_{R} \psi\left(\frac{x}{y}\right) \frac{d x}{|x|} \\
& =-\mu(\{0\}) \int_{R} \psi(s) d s+\int_{R} \psi(s) d s \int_{R} d \mu(t) .
\end{aligned}
$$

This shows that $S \mu \in L^{1}(R)$ and $T \hat{\mu}(0)=\mu(\{0\}) \int_{R} \psi(s) d s+(S \mu)(0)$ and establishes (b) for $x=0$. Similarly, for $x \neq 0$ and with the change of variable 
$y=(x / t) s, d y=(|x| /|t|) d s$, we get

$$
\begin{aligned}
T \hat{\mu}(x)-\mu(\{0\}) \int_{R} \psi(s) d s & =\int_{R-\{0\}} e^{-i x t} d \mu(t) \int_{R} \psi\left(\frac{y}{x}\right) \frac{d y}{|x|} \\
& =\int_{R-\{0\}} d \mu(t) \int_{R} e^{-i x s} \psi\left(\frac{s}{t}\right) \frac{d s}{|t|}=(S \mu) \hat{(x),}
\end{aligned}
$$

which is $(b)$ for $x \neq 0$.

Theorem 2. Let $\mu$ be a continuous measure in $M(R), \mu(R)=0$, and

$$
\dot{\mu}(x)=\int_{R} \frac{e^{-i x t}-1}{t} d \mu(t)=0
$$

for $0 \neq x \in R$. Then $\mu$ is the zero measure.

Proof. Let $T \hat{\mu}$ be the Hausdorff mean whose kernel $\psi$ is the characteristic function of $[0,1]$. Then $\dot{\mu}(x) /(-i x)=T \hat{\mu}(x)=0$ for $x \neq 0$. By Theorem $1, \mu(\{0\})=0$ and $\dot{\mu}(x)=-i x(S \mu) \hat{(x)}$ for $x \neq 0$. Since $T \hat{\mu}(0)=\hat{\mu}(0)=\mu(R)$

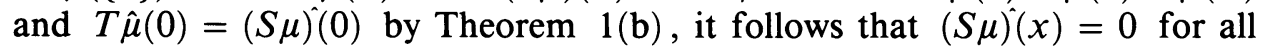
$x \in R$. Furthermore, if $E_{y}=[0, y]$ for $y>0$ and $E_{y}=[y, 0]$ for $y<0$, then it is easy to verify that

$$
\mu\left(E_{y}\right)=\int_{0}^{y} S \mu(x) d x-y \operatorname{sign}(y) S \mu(y) .
$$

Therefore, if $\mu$ is a continuous measure, then $S \mu(y)$ is continuous for $y \neq$ 0 and its Fourier integral is Cesaro summable to $S \mu(y)$ for $y \neq 0$. Since $(S \mu)(x)=0$, it follows that $S \mu(y)=0$ for $y \neq 0$. Hence, $\mu\left(E_{y}\right)=0$ for $y \in R$ because of equation (1) and the fact that $\mu(\{0\})=0$. Therefore, $\mu$ is the zero measure.

Theorem 3. Let $T \hat{\mu}$ be the Hausdorff mean of the Fourier-Stieltjes transform of measure $\mu \in M(R)$, whose kernel $0 \not \equiv \psi \in L^{1}(R)$ is nonnegative, nonincreasing, bounded, and has support in $[0, \infty)$. Then $-i \operatorname{sign}(x) T \hat{\mu}(x)$ is the Fourier transform of a function in $L^{1}(R)$ if and only if $T \hat{\mu}$ tends to zero at infinity and $x^{-1} \mu((-x, x)) \in L^{1}(0, \infty)$.

Proof. First we show that $-i \operatorname{sign}(x) T \hat{\mu}(x)$ is the Fourier transform of a function in $L^{1}(R)$ if and only if $\mu(\{0\})=0$ and the Hilbert transform of $S \mu$ belongs to $L^{1}(R)$. Suppose $-i \operatorname{sign}(x) T \hat{\mu}(x)=\hat{g}(x)$ for $g \in L^{1}(R)$. Then both $T \hat{\mu}(x)$ and $(S \mu)(x)$ tend to zero as $|x| \rightarrow \infty$ by the Riemann-Lebesgue theorem and $\mu(\{0\})=0$ by Theorem $1(\mathrm{~b})$ and the fact that $\psi \geq 0$. Moreover, $-i \operatorname{sign}(x) T \hat{\mu}(x)=-i \operatorname{sign}(x)(S \mu)(x)$. Thus, the Fourier integral of $-i \operatorname{sign}(x) T \hat{\mu}(x)$ is Abel summable to $g(x)$ a.e. [2, p.192], and at the same time it is Abel summable to the Hilbert transform $\widetilde{S} \mu(x)$ of the function $S \mu(x)$ a.e. [2, p.327]. Therefore, $(\widetilde{S} \mu)(x)=g(x)$ a.e. and $S \mu \in L^{1}(R)$. Conversely, if $T \hat{\mu}$ tends to zero at infinity and $\widetilde{S} \mu \in L^{1}(R)$, then $T \hat{\mu}=(S \mu)^{\hat{~}}$ by Theorem $1(\mathrm{~b})$ and $(S \mu)(x)=-i \operatorname{sign}(x)(S \mu)(x)$ [2, p.324]. In order to complete the proof of the theorem, it remains to show that $\widetilde{S} \mu \in L^{1}(R)$ if and only if $x^{-1} \mu((-x, x)) \in L^{1}(0, \infty)$, assuming that $\mu(\{0\})=0$.

(a) Let $\rho \in M(R)$ be an odd measure in $M(R)$. We will show that $\widetilde{S} \rho \in L^{1}(R)$. $S \rho(x)$ is an odd function in $L^{1}(R)$ and nonincreasing for $x>0$. Its Hilbert 
transform is even and for $x>0$

$$
\begin{aligned}
-\pi \tilde{S} \rho(x) & =\int_{-\infty}^{\infty} \frac{S \rho(t)}{t-x} d t=\left(\int_{3 x / 2}^{\infty}+\int_{x / 2}^{3 x / 2}+\int_{0}^{x / 2}\right)\left(\frac{1}{t-x}+\frac{1}{t+x}\right) S \rho(t) d t \\
& =g_{1}(x)+g_{2}(x)+g_{3}(x) .
\end{aligned}
$$

Thus, it suffices to show that $\widetilde{S} \rho \in L^{1}(0, \infty)$, assuming without loss of generality that $\mu \geq 0$ on $[0, \infty)$. Let $\varphi(x)=S(S \rho)(x)$ for $x>0$ when the kernel of $S$ is the characteristic function of $[0,1]$. Then $\varphi \in L^{1}(0, \infty)$ by Theorem 1(a). Now for $0<3 x / 2 \leq t<\infty, 1 /(t-x) \leq 3 / t, 1 /(t+x) \leq 1 / t$, $\left|g_{1}(x)\right| \leq(18 / 5) \varphi(3 x / 2)$, and $g_{1} \in L^{1}(0, \infty)$. Next

$$
g_{2}(x)=\int_{x / 2}^{3 x / 2} \frac{S \rho(t)}{t-x} d t+\int_{x / 2}^{3 x / 2} \frac{S \rho(t)}{t+x} d t=g_{4}(x)+g_{5}(x),
$$

where $\left|g_{5}(x)\right| \leq \phi(x / 2)$ and $g_{5} \in L^{1}(0, \infty)$. Since $S \rho(t) \geq 0$ and is nonincreasing for $t>0$, we have

$$
\begin{aligned}
\left|g_{4}(x)\right| & =\int_{0}^{x / 2}[S \rho(x-u)-S \rho(x+u)] \frac{d u}{u} \\
& =\int_{0}^{x / 2} \frac{d u}{u} \int_{[x-u, x)} \psi\left(\frac{x}{t}\right) \frac{d \rho(t)}{t}+\int_{0}^{x / 2} \frac{d u}{u} \int_{[x, x+u)} \psi\left(\frac{x}{t}\right) \frac{d \rho(t)}{t} \\
& \leq\|\psi\|_{\infty} \int_{[x / 2, x)} \log \frac{x}{2(x-t)} \frac{d \rho(t)}{t}+\|\psi\|_{\infty} \int_{[x, 3 x / 2)} \log \frac{x}{2(t-x)} \frac{d \rho(t)}{t} \\
& =\|\psi\|_{\infty} \rho((0, \infty))\left[\int_{1}^{2} \log \frac{s}{2(1-s)} d s+\int_{1 / 3}^{1} \log \frac{s}{2(1-s)} d s\right]<\infty .
\end{aligned}
$$

Thus, $g_{4} \in L^{1}(0, \infty)$, and $g_{2} \in L^{1}(0, \infty)$. Finally,

$$
\begin{aligned}
g_{3}(x) & =\int_{0}^{x / 2}\left(\frac{1}{x}+\frac{1}{t-x}\right) S \rho(t) d t+\int_{0}^{x / 2}\left(\frac{1}{t+x}-\frac{1}{x}\right) S \rho(t) d t \\
& =g_{6}(x)+g_{7}(x)
\end{aligned}
$$

Then $g_{6} \in L^{1}(0, \infty)$ and $g_{7} \in L^{1}(0, \infty)$ because

$$
\begin{aligned}
& \left|g_{6}(x)\right| \leq \frac{2}{x^{2}} \int_{0}^{x / 2} t S \rho(t) d t=\omega(x), \quad\left|g_{7}(x)\right| \leq \frac{1}{2} \omega(x), \\
& \int_{0}^{\infty} \omega(x) d x=\int_{0}^{\infty} t S \rho(t) d t \int_{2 t}^{\infty} \frac{2}{x^{2}} d x=\int_{0}^{\infty} S \rho(t) d t<\infty .
\end{aligned}
$$

This implies that $g_{3} \in L^{1}(0, \infty)$. Hence $\tilde{S} \rho \in L^{1}(R)$.

(b) Let $\sigma$ be an even measure in $M(R)$. We show that $\tilde{S} \sigma \in L^{1}(R)$ if and only if $x^{-1} \sigma((0, x)) \in L^{1}(0, \infty)$. We may assume $\sigma \geq 0$ on $[0, \infty)$. $S \sigma(x)$ is an even function in $L^{1}(R)$ and nonincreasing for $x>0$; its Hilbert transform is odd and for $x>0$,

$$
\begin{aligned}
-\pi \widetilde{S} \sigma(x) & =\left(\int_{3 x / 2}^{\infty} \int_{x / 2}^{3 x / 2}+\int_{0}^{x / 2}\right)\left(\frac{1}{t-x}-\frac{1}{t+x}\right) S \sigma(t) d t \\
& =f_{1}(x)+f_{2}(x)+f_{3}(x) .
\end{aligned}
$$


The argument that showed $g_{1} \in L^{1}(0, \infty)$ in part (a) also shows that $f_{1} \in$ $L^{1}(0, \infty)$. Put

$$
f_{2}(x)=\int_{x / 2}^{3 x / 2} \frac{S \sigma(t)}{t-x} d t-\int_{x / 2}^{3 x / 2} \frac{S \sigma(t)}{t+x} d t=f_{4}(x)-f_{5}(x) .
$$

The proof that $f_{4}, f_{5} \in L^{1}(0, \infty)$ is identical with that given for $g_{4}, g_{5} \in$ $L^{1}(0, \infty)$ in $(\mathrm{a})$. Finally, let

$$
\begin{aligned}
f_{3}(x)= & \int_{0}^{x / 2}\left(\frac{1}{x}+\frac{1}{(t-x)}\right) S \sigma(t) d t-\int_{0}^{x / 2}\left(\frac{1}{t+x}-\frac{1}{x}\right)-S \sigma(t) d t \\
& -\frac{2}{x} \int_{0}^{x / 2} S \sigma(t) d t \\
= & f_{6}(x)-f_{7}(x)-\theta\left(\frac{x}{2}\right) .
\end{aligned}
$$

Here, $f_{6}, f_{7} \in L^{1}(0, \infty)$ for the same reason that $g_{6}, g_{7} \in L^{1}(0, \infty)$ in (a). Thus, $f_{3}$ differs from an integrable function on $(0, \infty)$ by the function $-\theta(x)$. But

$$
\begin{aligned}
\theta(x) & \geq \frac{1}{x} \int_{0}^{x} d u \int_{u}^{\infty} \psi\left(\frac{u}{t}\right) \frac{d \sigma(t)}{t}=x^{-1} \sigma((0, x)) \int_{0}^{1} \psi(s) d s, \\
\theta(x) & =\frac{1}{x} \int_{0}^{x} d u \int_{0}^{x} \psi\left(\frac{u}{t}\right) \frac{d \sigma(t)}{t}+\frac{1}{x} \int_{0}^{x} d u \int_{x}^{\infty} \psi\left(\frac{u}{t}\right) \frac{d \sigma(t)}{t} \\
& \leq x^{-1} \sigma((0, x))\|\psi\|_{1}+\|\psi\|_{\infty} S \sigma(x),
\end{aligned}
$$

by Fubini's theorem. From the preceding inequalities it follows that $\theta \in$ $L^{1}(0, \infty)$ if and only if $x^{-1} \sigma((0, x)) \in L^{1}(0, \infty)$.

Finally, let $\mu \in M(R)$ and $2 \rho(E)=\mu(E)+\mu(-E)$ and $2 \sigma(E)=\mu(E)+$ $\mu(-E)$. Then $\rho$ is an odd measure, $\sigma$ is an even measure, and $\widetilde{S} \mu=\widetilde{S} \rho+\widetilde{S} \sigma$. By (a) $\widetilde{S} \rho \in L^{1}(R)$, and by (b) $\widetilde{S} \sigma \in L^{1}(R)$ if and only if $x^{-1} \sigma((0, x)) \epsilon$ $L^{1}(0, \infty)$, or equivalently $x^{-1} \mu((-x, x)) \in L^{1}(0, \infty)$, and thus completes the proof of Theorem 3 .

Corollary 1. For $\psi \in L^{1}(R), \mu \in M(R)$, and $p \in R$, let

$$
\begin{gathered}
r(x)=T\left(\hat{\mu} \cdot \hat{\delta}_{p}\right)(x)-\mu(\{p\}) \int_{R} \psi(s) d s, \\
R(x)=T|\hat{\mu}|^{2}(x)-\sum_{p \in R}|\mu(\{p\})|^{2} \int_{R} \psi(s) d s .
\end{gathered}
$$

Then

(a) $r(x)$ and $R(x)$ are Fourier transforms of functions in $L^{1}(R)$.

(b) $-i \operatorname{sign}(x) r(x)$ is the Fourier transform of a function in $L^{1}(R)$ if and only if $T\left(\hat{\mu}, \hat{\delta}_{p}\right)$ tends to zero at infinity and $x^{-1} \mu((-p-x,-p+x)) \in L^{1}(R)$, provided $0 \not \equiv \psi \geq 0$, is nonincreasing, bounded, and has support in $[0, \infty)$.

(c) $-i \operatorname{sign}(x) R(x)$ is the Fourier transform of a function in $L^{1}(R)$ if and only if $T|\hat{\mu}|^{2}$ tends to zero at infinity and $x^{-1} \mu * \mu^{*}((-x, x)) \in L^{1}(R)$, provided $\psi$ satisfies the conditions for (b). 
Corollary 1 follows from Theorems 1 and 3 when applied to the convolution $\mu * \delta_{p}$, where $\delta_{p}$ is the unit mass at the point $p$, and to $\mu * \mu^{*}$, where $\mu^{*}(E)=\overline{\mu(-E)}$. When $\psi$ is the characteristic function of $[0,1]$ and $\mu$ is replaced by the even part of $\mu$, given by $2 \sigma(E)=\mu(E)+\mu(-E)$, the Hausdorff mean reduces to the integral arithmetic average and Corollary 1 provides a considerable improvement of the Theorems of Fejer and Wiener [10, pp. 102-104].

Finally, it is of interest to note that Corollary 1 yields a strengthened generalization of the mean ergodic theorem for the Hausdorff mean of a continuous parameter group of unitary operators. Consider a one-parameter group $U_{t}$, $t \in R$, of unitary operators on a Hilbert space $X$ such that $\left(U_{t} f, g\right)$ is continuous for $f, g \in X$. By Stone's theorem

$$
U_{t}=\int_{R} e^{-i t \lambda} d E(\lambda),
$$

where $E(\lambda)$ a projection-valued spectral measure on $R$. Moreover, $(E(\lambda) f, g)$ is a regular bounded complex Borel measure on $R$ whose total variation is $\leq\|f\| \cdot\|g\|$ and Fourier-Stieltjes transform is given by $\left(U_{t} f, g\right)$ and $\|E(\lambda) f\|^{2}$ is a positive regular Borel measure on $R$ with total variation $\|f\|^{2}$ (see [13, pp. 381-389]). Let $\psi$ be continuous in $L^{1}(R)$, and consider the Hausdorff mean $M_{x}^{(a)} f$ of the vector-valued function $U_{a+t} f, f \in X$, given by

$$
M_{x}^{(a)} f=\frac{1}{|x|} \int_{R} \psi\left(\frac{t}{x}\right) U_{a+t} f d t, \quad 0 \neq x \in R, a \in R,
$$

defined as a limit of Riemann sums, and so that $M_{0}^{(a)} f=\left(\int_{R} \psi(s) d s\right) U_{a} f=$ $\bar{\psi} U_{a} f$. It is easy to see that

$$
\hat{\delta}_{\lambda}(a) T \hat{\delta}_{\lambda}(x)-\delta_{\lambda}(\{0\}) \bar{\psi}=\hat{\delta}_{\lambda}(a)\left[T \hat{\delta}_{\lambda}(x)-\delta_{\lambda}(\{0\}) \bar{\psi}\right]
$$

for $\lambda, a \in R$ and that it is convergent to zero as $|x| \rightarrow \infty$ by Corollary $1(a)$. In fact, the convergence is uniform for $p \in R$ and bounded by $\|\psi\|_{1}+|\bar{\psi}|$ for $\lambda \in R$. Therefore,

$$
\begin{gathered}
M_{x}^{(a)}-\bar{\psi} E(\{0\})=\int_{R}\left(\hat{\delta}_{\lambda}(a) T \hat{\delta}_{\lambda}(x)-\delta(\{0\}) \bar{\psi}\right) d E(\lambda), \\
\left\|M_{x}^{(a)} f-\bar{\psi} E(\{0\}) f\right\|^{2}=\int_{R}\left|\hat{\delta}_{\lambda}(a) T \hat{\delta}_{\lambda}(x)-\delta_{\lambda}(\{0\}) \bar{\psi}\right|^{2} d\|E(\lambda) f\|^{2},
\end{gathered}
$$

which tends to zero as $|x| \rightarrow \infty$ uniformly for $p \in R$ and yields part of Corollary 2 below. The rest is simply a restatement of Corollary 1(a), because as noted above $\left(U_{t} f, g\right)$ is the Fourier-Stieltjes transform of the measure $(E(\lambda) f, g)$ in $M(R)$ and

$$
\left(\frac{1}{|x|} \int_{R} \psi\left(\frac{t}{x}\right) U_{t} f d t-\bar{\psi} E(\{0\}) f, g\right)=r(x) .
$$

Note that when $\psi$ is the characteristic function of $[0,1]$, Corollary 2 reduces to von Neumann's mean ergodic theorem (see [3, p. 389]), and when $\bar{\psi}=0$, Corollary 2 says that the Hausdorff mean of $U_{a+t}$ converges strongly to zero. 
Corollary 2. Let $U_{t}$ be a one-parameter continuous group of unitary operators for $t \in R$. Then the Hausdorff mean of $U_{a+t}$ with a continuous $L^{1}$-kernel converges strongly to a scalar multiple of a projection, and for $a=0$ their difference tends weakly to zero at the rate of a Fourier transform of an $L^{1}$-function as in Corollary $1(\mathrm{a})$.

\section{REFERENCES}

1. R. P. Boas Jr., Integrability theorems for trigonometric transforms, Springer-Verlag, New York, 1967.

2. P. L. Butzer and R. J. Nessel, Fourier analysis and approximation, Vol. 1, Birkhauser-Verlag, Basel, 1971.

3. E. Fabes, M. Jodeit, and J. F. Lewis, On the spectra of a Hardy kernel, J. Funct. Anal. 21 (1976), 187-194.

4. C. Georgakis, On the arithmetic mean of Fourier-Stieltjes coefficients, Proc. Amer. Math. Soc. 33 (1972), 467-484.

5. R. R. Goldberg, Certain operators and Fourier transforms on $L^{2}$, Proc. Amer. Math. Soc. 10 (1959), 385-390.

6. C_ Convolutions and general transforms on $L^{p}$, Duke Math. J. 27 (1960), 251-259.

7. G. Goes, Arithmetic means of Fourier-Stieltjes sine coefficients, Proc. Amer. Math. Soc. 14 (1963), 10-11.

8. G. H. Hardy, Divergent series, Clarendon Press, Oxford, 1949.

9. G. H. Hardy, J. E. Littlewood, and G. Polya, Inequalities, Cambridge Univ. Press, London and New York, 1934.

10. T. Kawata, Fourier analysis in probability theory, Academic Press, New York, 1972.

11. G. Leibowitz, A convolution approach to Hausdorff integral operators, Indiana J. Math. 23 (1973), 447-460.

12. B. E. Rhoades, Spectra of some Hausdorff operators, Acta. Sci. Math. (Szeged) 32 (1972), 91-100.

13. F. Riesz and B. Sz. Nagy, Functional analysis, Ungar, New York, 1955.

14. I. Schur, Bemerkungen zur theorie des Beschränkten billinear formen mit unendlich Vieben Verändlichen, J. Reine Angew. Math. 140 (1911), 1-27.

Department of Mathematics, DePaul University, Chicago, Illinois 60614

E-mail address: MATCG@orion.DePaul.Edu. 